\title{
Primitive Anorectal Malignant Melanoma: A Rare But Aggressive Tumor
}

\author{
Asmaa Elkarouachi, Ahmed Ballati, Asmar Mohamed, Saâd Rifki Jai, Driss Erguibi, Rachid Boufettal, \\ and Farid Chehab
}

\begin{abstract}
We report 2 observations of primary anorectal melanoma, collected over a period of 2 years from December 2017 to December 2019, as well as data from the literature. The patients nevertheless benefited after the histological evidence with an additional immunohistochemical study of an extension assessment made by pelvic MRI and thoracoabdominopelvic CT scan. The patients were classified stage I according to the Slingluff classification. They underwent abdominoperineal resection with definitive left iliac colostomy and adjuvant Chemotherapy.
\end{abstract}

Index Terms-Abdominoperineal resection; melanoma; prognosis; rectum; wide local excision.

\section{INTRODUCTION}

The primary anorectal melanoma remains a very rare entity, less than $2 \%$ of melanomas, and is third after the skin and retina [1]. This tumour has a slight female predominance and occurs mainly between the sixth and seventh decade [2].The cornerstone of the treatment remains surgery, therefore two options are available to the surgeon which are local resection or abdominoperineal resection, whose results in terms of survival remain almost comparable, but the latter offers better local control of the disease.The prognosis is poor because patients present in advanced stages due to the high metastatic potential. Through our case study we will clarify the clinical and therapeutic characteristics.

\section{PATIENTS AND OBSERVATIONS}

\section{A. Case 1}

This is an 86 year old patient, a chronic smoker, who presented a rectal syndrome, proctalgia and rectorragies of low abundance; all of which evolved over a period of 5 months in a context of weight loss of $12 \mathrm{~kg}$ and an alteration of the general state. Somatic examination: Performance status 1, body mass index $18 \mathrm{~kg} / \mathrm{m} 2$, proctological examination reveals an ulcerating hard tumour extending into the canal up to $15 \mathrm{~cm}$. The tumour was biopsied and an objective anatomopathological examination showed a poorly

differentiated malignant tumour proliferation requiring immunohistochemical supplementation which showed a positivity of PS 100, Melan A and HMB 45 thus concluding to an undifferentiated large cell infiltrating tumour proliferation compatible with an achromic melanoma.As part of the extension work-up, a colonoscopy that did not

Published on April 09, 2020.

Authors are with Ibn Rochd University Hospital, Casablanca, Morocco. (corresponding e-mail: ahmedballati@gmail.com) reveal any other lesions and a thoracoabdominopelvic CT scan that revealed an $18 \mathrm{~mm}$ thickening of the lower rectum with normal perirectal fat and no other locoregional or

secondary localizations were found.Pelvic MRI (Figure 1) showed a thickening of the lower and middle rectum coming into lateral contact with the fascia recti and invading the anal canal and sphincters at the bottom. The 18fluorodeoxyglucose PET-CT (Figure 2) showed a hypermetabolic process of the anal canal and lower rectum SUV max 7.51 metabolic volumes $70.85 \mathrm{~cm} 3$ extended over $10 \mathrm{~cm}$. At the end of this assessment, the tumour was classified as stage I according to the Slingluff classification. The patient had an extralevatorial abdominoperineal resection with definitive iliac colostomy. Histological examination of the specimen, with a neoplasm of $9 * 8 \mathrm{~cm}$, revealed a malignant tumour proliferation of the round-cell rectum compatible with melanoma.

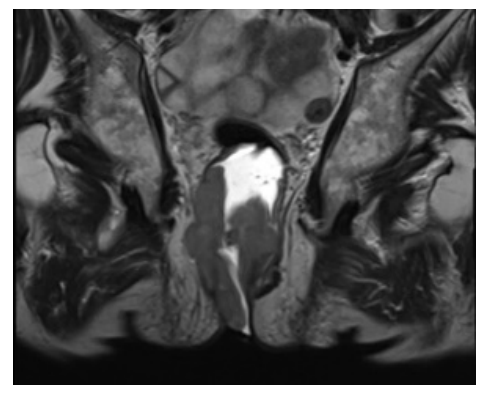

Fig. 1. Thickening of the lower and middle rectum on a pelvic MRI.

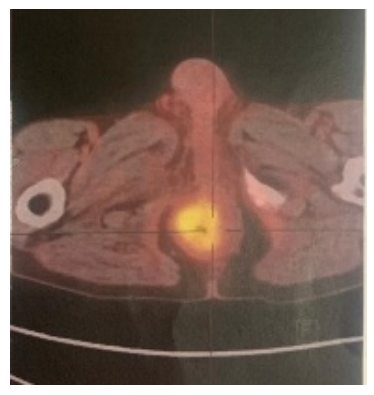

Fig. 2. Hyperfixation of lower rectum on scintigraphy.
An immunohistochemical study was completed which showed tumour proliferation expressing PS100, HMB 45 and Melan A but not expressing CKAE1, AE 3, Ckit, chromogranin or CD 45, therefore the diagnosis of achromic malignant melanoma of the rectum was retained with a tumour thickness of $14 \mathrm{~mm}$ and sixteen lymph nodes were removed from the mesorectum, two of which were positive. The final classification of this melanoma was pT3N1 according to the classification of the American Joint Committee on Cancer, and further treatment with chemotherapy was indicated and 12 months after surgery the patient is free of recurrence.

\section{B. Case 2}

He is a 34 year old patient with no particular pathological history and has been suffering from chronic constipation with intermittent rectorragies for 3 years. The patient 
consulted a gastroenterologist for hemorrhoids. And during the proctological examination, an ulcerating ulcerating anal tumour measuring $4 \mathrm{~cm}$ in major axis, of stony consistency, blackish in colour and no inguinal adenopathies is discovered during the rectal examination. An anatomopathological examination of a biopsy fragment of the tumour is in favour of an achromic malignant melanoma. A thoracoabdominopelvic CT scan was requested as part of the extension work-up revealing a tumour process from the anal canal extended to the lower rectum by $64 \mathrm{~mm}$, stenosing, developed essentially at the expense of the right anterolateral surface and responsible for a stenosis of the digestive lumen essentially distally.

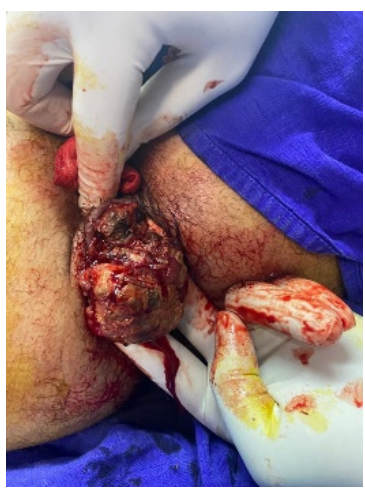

Fig. 3. Intraoperative view of the tumour prolapsed by the anus.

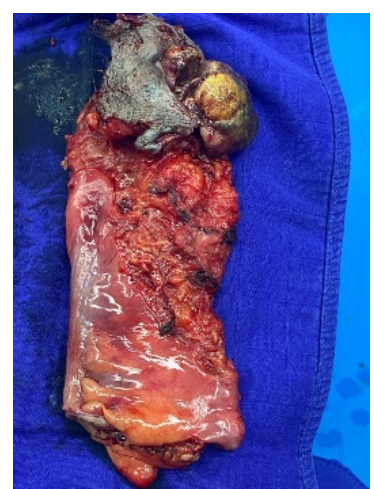

Fig. 4 .Abdominoperineal resection specimen.
It is in intimate contact with the right posterolateral border of the prostate, partially invades the right anus elevator muscle and the right internal sphincter associated with micronodules of the largest right mesorectum $(8.6 \mathrm{~mm})$ without hepatic or pulmonary metastasis. At the end of this assessment, the tumour is classified as stage I according to the Slingluff classification. The patient was operated on and underwent abdominoperineal resection (Figure 3 and 4). The postoperative aftereffects were simple and the patient was declared out sick at D7 postoperatively. The results of the anatomopathological examination showed a poorly differentiated tumour proliferation, largely necrotic with melanic pigments and the further immunohistochemical study was in favour of a malignant melanoma expressing PS 100 (Figure 5), HMB 45 (Figure 6) and Melan A (Figure 7) with 2 lymph node metastases out of 22 samples, supplemented by adjuvant chemotherapy. The patient presented a local recurrence and benefited from palliative radiotherapy with analgesic aim.

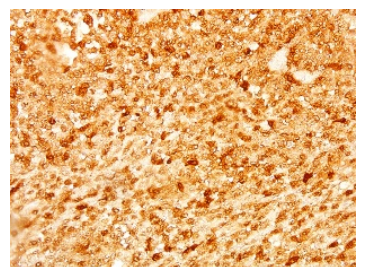

Fig. 5. Tumour cells express PS100.

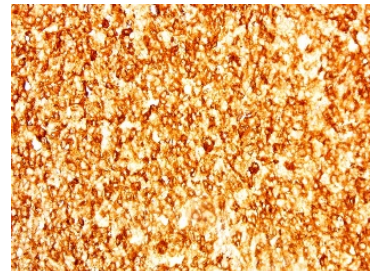

Fig. 6. Tumour cells express HMB 45

\section{DISCUSSION}

Primary anorectal melanoma, as its name suggests, develops from melanocytes, which are present in squamous epithelium of the anal canal. The clinical symptoms of anorectal melanoma are essentially: rectorragies, anal pain and a perianal mass, rectal syndrome, inguinal mass related to inguinal lymphadenopathy. Due to the non-specificity of the symptomatology, $60 \%$ of patients were already metastatic at the time of diagnosis [3]. Macroscopically, primary anorectal melanoma frequently presents as an ulcerovegetant or polypoid, pedicled lesion, and in half of the cases is black or amelanic in colour [4]. Rectal localizations of the disease are often tumors that have developed at the expense of the anal canal and have subsequently infiltrated the rectal wall, to be differentiated in its macroscopic appearance from hemorrhoids or polyp. The diagnosis is histologically based on the detection of melanin pigment within the tumour cells. In the amelanic forms, immunohistochemical study is essential, allowing to highlight a positive immunostaining for the $\mathrm{S}-100$ protein, vimentin and melanoma specific antibodies: HMB 45 [4] .In our study the complement of immunohistochemical to PS 100, HMB 45 and Melan A was required. Because of the frequency of digestive metastases of melanoma, the primitive character should not be evoked first. Pelvic computed tomography is a good reference for monitoring progress and looking for pelvic recurrences[5]. Our patients all had a thoracoabdominal pelvic CT scan to evaluate locoregional and remote extension. Node involvement affects first the inguinal and lower mesenteric chains and secondarily the para-aortic chains. Given the rarity of anorectal melanoma, its optimal treatment remains controversial. Several classifications of primary anorectal melanoma have been proposed. The Slingluff classification classifies melanoma into 3 progressive stages [6].In study, the 2 patients were classified as stage I. There is a second Breslow classification, consisting of 5 stages depending on the depth of invasion, which is of prognostic interest. Surgery is the treatment of choice for primary anorectal melanomas, but its modalities of removal, either localized or abdominoperineal resection, remain controversial [7]. Initially, studies have proposed abdominoperineal resection combined with pelvic lymph node dissection, giving better results for primary anorectal melanoma. In our study, patients benefited from abdominoperineal resection because of the intimate contact with the sphincter apparatus by contrast, other studies have shown that local resection without lymphadenectomy gives similar results in terms of recurrence [8]. A Japanese meta-analysis conducted in 2015 by Matsuda et al. concluded that abdominoperineal resection has no survival benefit for the patient. Since local failure after local resection could be managed by salvage surgery, 
prophylactic lymph node dissection is indicated only if pelvic adenopathies are present [9]. The efficacy of chemotherapy has not been proven [10]. The role of radiotherapy also remains limited. There is a second Breslow classification, consisting of 5 stages depending on the depth of invasion, which is of prognostic interest. Surgery is the treatment of choice for primary anorectal melanomas, but its modalities of removal, either localized or abdominoperineal resection, remain controversial [7]. Initially, studies have proposed abdominoperineal resection combined with pelvic lymph node dissection, giving better results for primary anorectal melanoma. In our study, patients benefited from abdominoperineal resection because of the intimate contact with the sphincter apparatus by contrast, other studies have shown that local resection without lymphadenectomy gives similar results in terms of recurrence [8].A Japanese meta-analysis conducted in 2015 by Matsuda et al. concluded that abdominoperineal resection has no survival benefit for the patient. Since local failure after local resection could be managed by salvage surgery, prophylactic lymph node dissection is indicated only if pelvic adenopathies are present [9]. The efficacy of chemotherapy has not been proven [10]. The role of radiotherapy also remains limited.

\section{CONCLUSION}

Anorectal malignant melanomas still has a very poor prognosis with a short survival time, due to diagnostic delay and high malignant potential. There is still no clear consensus in this form of anal tumour. The basis of treatment is surgical, chemotherapy and radiotherapy are still under discussion and new therapeutic procedures such as targeted therapies may emerge.

\section{REFERENCES}

[1] M. M. Melhouf, N. el Amrani, H. Mathieu-Daude, et J. B. Dubois, « Les mélanomes malins anorectaux. A propos de 5 cas: revue de la littérature. ", Ann Gastroenterol Hepatol Paris, p. 209 12, 1995.

[2] C. Falch et al., " Anorectal malignant melanoma: extensive 45-year review and proposal for a novel staging classification », J. Am. Coll. Surg., vol. 217, no 2, p. 324 335, août 2013.

[3] A. da C. Veloso, J. C. C. Magno, et J. A. D. da C. e Silva, « Anal melanoma: a rare, but catastrophic tumor ", J. Coloproctology, vol. 34, no 1, p. 9 13, janv. 2014.

[4] J. L. Canales et J. M. G. Blesa, « Amelanotic Anorectal Malignant Melanoma: Case Report with Immunohistochemical Study and Literature Review », in Case reports in oncology, 2009.

[5] S. Rohr, H. Sadok, B. Dai, et C. Meyer, "[Anorectal malignant melanomas. Apropos of 2 new cases] », J. Chir. (Paris), vol. 129, no 6 7, p. 320 323, juill. 1992

[6] « Clinical Practice Guidelines for the Management of Melanoma in Australia and New Zealand », p. 268.

[7] T. Takahashi et al., " Anorectal melanoma: report of three cases with extended follow-up », South. Med. J., vol. 97, no 3, p. 311 313, mars 2004.

[8] C. Thibault, P. Sagar, S. Nivatvongs, D. M. Ilstrup, et B. G. Wolff, « Anorectal melanoma--an incurable disease? », Dis. Colon Rectum, vol. 40, no 6, p. 661668 , juin 1997.

[9] A. Matsuda et al., « Abdominoperineal resection provides better local control but equivalent overall survival to local excision of anorectal malignant melanoma: a systematic review », Ann. Surg., vol. 261, no 4, p. 670677 , avr. 2015.

[10] K. B. Kim et al., "Biochemotherapy in patients with metastatic anorectal mucosal melanoma », Cancer, vol. 100, no 7, p. 1478 1483, 2004.
Asma Elkarouachi

20240, Casablanca,Morocco

Telephone :00212614110017

e-mail: dr.elkarouachi@gmail,com

Institution: Department of General Surgery III, Ibn Rochd University

Hospital, Casablanca, Morocco.

\section{Ahmed Ballati}

e-mail: ahmedballati@gmail,com

Institution: Department of General Surgery III, Ibn Rochd University Hospital, Casablanca, Morocco.

\section{Mohamed Asmar}

e-mail: asmar.med87@gmail.com

Institution: Department of General Surgery III, Ibn Rochd University Hospital, Casablanca, Morocco.

\section{Saad Rifki El Jai}

e-mail: Saadjai@yahoo.fr

Institution: Department of General Surgery III, Ibn Rochd University Hospital, Casablanca, Morocco.

\section{Rachid Boufettal}

e-mail: racbouf@hotmail.fr

Institution: Department of General Surgery III, Ibn Rochd University Hospital, Casablanca, Morocco.

\section{Driss Errguibi}

e-mail: erguibi_d@yahoo.fr

Institution: Department of General Surgery III, Ibn Rochd University Hospital, Casablanca, Morocco.

\section{Farid Chehab}

e-mail:Farid_chehab@yahoo.fr

Head of the digestive cancer and liver transplant surgery department Institution: Department of General Surgery III, Ibn Rochd University Hospital, Casablanca, Morocco. 